\title{
Improved Upper Bounds for the Laplacian Spectral Radius of a Graph
}

\author{
Tianfei Wang ${ }^{1}$ \\ ${ }^{1}$ School of Mathematics and Information Science \\ Leshan Normal University, Leshan 614004, P.R. China \\ $1_{\text {wangtf818@sina.com }}$ \\ Jin Yang ${ }^{2}$, Bin $\mathrm{Li}^{3}$ \\ ${ }^{2,3}$ Laboratory of Intelligent Information Processing and Application \\ Leshan Normal University, Leshan 614004, P.R. China \\ ${ }^{2}$ jinnyang@163.com, ${ }^{3}$ zishan818@163.com \\ Submitted: May 16, 2010; Accepted: Jan 24, 2011; Published: Feb 14, 2011 \\ Mathematics Subject Classifications: O5C50, 15A18
}

\begin{abstract}
In this paper, we present three improved upper bounds for the Laplacian spectral radius of graphs. Moreover, we determine all extremal graphs which achieve these upper bounds. Finally, some examples illustrate that the results are best in all known upper bounds in some sense.
\end{abstract}

\section{Introduction}

Let $G$ be a simple connected graph with vertex set $V(G)=\left\{v_{1}, v_{2}, \cdots, v_{n}\right\}$ and edge set $E(G)=\left\{e_{1}, e_{2}, \cdots, e_{m}\right\}$. For any vertex $v \in V(G)$, the degree of $v$, the set of neighbors of $v$ and the average of the degrees of the vertices adjacent to $v$ are denoted by $d_{v}, N_{v}$ and $m_{v}$, respectively. Let $D(G)=\operatorname{diag}\left(d_{u}, u \in V(G)\right)$ and $A(G)=\left(a_{u v}\right)$ be the diagonal matrix of the vertex degrees and the $(0,1)$ adjacency matrix of $G$, respectively. Then the matrix $L(G)=D(G)-A(G)$ is called the Laplacian matrix of a graph $G$. Obviously, it is symmetric and positive semi-definite, and consequently its eigenvalues are nonnegative real numbers. In addition, since each row sum of $L(G)$ is 0,0 is the smallest eigenvalue of $L(G)$. Therefore, the eigenvalues of $L(G)$, which are called the Laplacian eigenvalues of $G$, can be denoted by $\lambda_{1}(G) \geqslant \lambda_{2}(G) \geqslant \cdots \geqslant \lambda_{n}(G)=0$, where $\lambda_{1}(G)(=\lambda(G))$ is also called the Laplacian spectral radius. Let $K(G)=D(G)+A(G)$. It is called the signless Laplacian matrix of $G$ (or Quasi-Laplacian). A semiregular bipartite graph $G=(V, E)$ is a graph with bipartition $\left(V_{1}, V_{2}\right)$ of $V$ such that all vertices in $V_{i}$ have the same degree $k_{i}$ 
for $i=1,2$. The Laplacian eigenvalues of a graph are important in graph theory, because they have close relations to numerous graph invariants, including connectivity, diameter, isoperimetric number, maximum cut, etc. The study of the Laplacian spectrum of graphs has attracted much research interest in recent years. Particularly, good upper bounds for $\lambda(G)$ are needed in many applications [1-5]. Apart from some early bounds, most of them emerged from 1997.

In 1997, Li and Zhang [6] described an upper bound as follows

$$
\lambda(G) \leqslant 2+\sqrt{(r-2)(s-2)},
$$

where $r=\max \left\{d_{u}+d_{v}: u v \in E(G)\right\}$, and if $r=d_{p}+d_{q}$, then $s=\max \left\{d_{x}+d_{y}: x y \in\right.$ $E(G)-\{p q\}\}$.

In 1998, Merris [7] showed that

$$
\lambda(G) \leqslant \max \left\{d_{u}+m_{u}: u \in V(G)\right\} .
$$

At the same time, Li and Zhang [8] reported the following result, which is an improvement of (2):

$$
\lambda(G) \leqslant \max \left\{\frac{d_{u}\left(d_{u}+m_{u}\right)+d_{v}\left(d_{v}+m_{v}\right)}{d_{u}+d_{v}}: u v \in E(G)\right\} .
$$

In 2000, Rojo, et al. [9], obtained the following bound:

$$
\lambda(G) \leqslant \max \left\{d_{u}+d_{v}-\left|N_{u} \cap N_{v}\right|: u \neq v\right\},
$$

where $\left|N_{u} \cap N_{v}\right|$ is the number of common neighbors of vertex $u$ and $v$.

In 2001, Li and Pan [10] proved that

$$
\lambda(G) \leqslant \max \left\{\sqrt{2 d_{u}\left(d_{u}+m_{u}\right)}: u \in V(G)\right\} .
$$

In 2003, K.C. Das [11] improved the bound (4) as follows

$$
\lambda(G) \leqslant \max \left\{d_{u}+d_{v}-\left|N_{u} \cap N_{v}\right|: u v \in E(G)\right\} .
$$

In 2004, K.C. Das [12] obtained another upper bound

$$
\lambda(G) \leqslant \max \left\{\frac{d_{u}+d_{v}+\sqrt{\left(d_{u}-d_{v}\right)^{2}+4 m_{u} m_{v}}}{2}: u v \in E(G)\right\} .
$$

In 2004, Zhang [13] proved that

$$
\lambda(G) \leqslant \max \left\{d_{u}+\sqrt{d_{u} m_{u}}: u \in V(G)\right\} .
$$


Furthermore, Zhang pointed out that (8) is always better than (5). Another bound was reported by the same author [13]

$$
\lambda(G) \leqslant \max \left\{\sqrt{d_{u}\left(d_{u}+m_{u}\right)+d_{v}\left(d_{v}+m_{v}\right)}: u v \in E(G)\right\} .
$$

In 2005, Guo [14] obtained a new upper bound for $\lambda(G)$, which improved Das's bound from [11], it reads:

$$
\lambda(G) \leqslant \max \left\{\frac{d_{u}+\sqrt{d_{u}^{2}+8 d_{u} m_{u}^{\prime}}}{2}: u \in V(G)\right\},
$$

where $m_{u}^{\prime}=\frac{\sum_{u v \in E(G)}\left(d_{v}-\left|N_{u} \bigcap N_{v}\right|\right)}{d_{u}}$.

Recently, in [5], we have obtained two further bounds:

$$
\begin{gathered}
\lambda(G) \leqslant \max \left\{\frac{d_{u}+d_{v}+\sqrt{\left(d_{u}-d_{v}\right)^{2}+4 \sqrt{d_{u} m_{u}} \cdot \sqrt{d_{v} m_{v}}}}{2}: u v \in E(G)\right\} . \\
\lambda(G) \leqslant 2+\max \left\{\sqrt{\frac{\left(d_{u}+d_{v}-2\right)\left(d_{u}^{2} \cdot m_{u}+d_{v}^{2} \cdot m_{v}-2 d_{u} d_{v}\right)}{d_{u} d_{v}}}: u v \in E(G)\right\} .
\end{gathered}
$$

In Section 2, we introduce some lemmas and some new and sharp upper bounds for $\lambda(G)$, which are better than all of the above mentioned upper bounds in some sense, and determine the extremal graphs which achieve these upper bounds. Some examples are presented in Section 3.

\section{Lemmas and results}

In the sequel, we denote by $\mu(M)$ the spectral radius of $M$, if $M$ is a nonnegative matrix. In order to obtain our main results, the following lemmas are required:

Lemma 2.1. ${ }^{[15]}$ Let $M=\left(m_{i j}\right)$ be an $n \times n$ irreducible nonnegative matrix with spectral radius $\mu(M)$, and let $R_{i}(M)$ be the $i$ th row sum of $M$, i.e $R_{i}(M)=\sum_{j=1}^{n} m_{i j}$. Then

$$
\min \left\{R_{i}(M) \mid 1 \leqslant i \leqslant n\right\} \leqslant \mu(M) \leqslant \max \left\{R_{i}(M) \mid 1 \leqslant i \leqslant n\right\} .
$$

Moreover, if the row sums of $M$ are not all equal, then both inequalities in (13) are strict.

Let $A=\left(a_{i j}\right) \in C^{n \times n}$ and $r_{i}=\sum_{j \neq i}\left|a_{i j}\right|$ for each $i=1,2, \ldots, n$, then

Lemma 2.2. ${ }^{[15]}$ Let $A=\left(a_{i j}\right)$ be a complex matrix of order $n \geqslant 2$. Then the eigenvalues of the matrix of $A$ lie in the region of the complex plane determined by the union of Cassini oval

$$
S_{i j}=\left\{z:\left|z-a_{i i}\right| \cdot\left|z-a_{j j}\right| \leqslant r_{i} r_{j}\right\},(i, j=1,2, \cdots, n ; i \neq j) .
$$


For convenience, let $\mu(K)(=\mu(K(G)))$ be the spectral radius of $K(G)$. The following lemma gave the relation between $\lambda(G)$ and $\mu(K)$.

Lemma 2.3. ${ }^{[16]}$ Let $G=[V, E]$ be a connected graph with $n$ vertices. Then $\lambda(G) \leqslant \mu(K)$, with equality if and only if $G$ is bipartite graph.

The line graph $H$ of a graph $G$ is defined by $V(H)=E(G)$, where any two vertices in $H$ are adjacent if and only if they are adjacent as edges of $G$. Denote the adjacency matrix of $H$ by $B$.

Lemma 2.4. ${ }^{[17]}$ Let $G$ be a simple connected graph and $\mu(B)$ be the largest eigenvalue of the adjacency matrix of the line graph $H$ of $G$. Then

$$
\lambda(G) \leqslant 2+\mu(B),
$$

with equality if and only if $G$ is bipartite graph.

Lemma 2.5. ${ }^{[6]}$ Let $A$ be an irreducible matrix which has two rows such that each of these rows contains at least two nonzero off-diagonal entries. If $\lambda$ lies on the boundary of $S=\bigcup_{i<j} S_{i j}$, then $\lambda$ is a boundary point of each $S_{i j}$.

Lemma 2.6. ${ }^{[16]}$ Let $G=(V, E)$ be a simple connected graph and $\lambda(G)$ be the largest Laplacian eigenvalue of $G$. If the equality in (3) holds, i.e

$$
\lambda(G)=\max \left\{\frac{d_{u}\left(d_{u}+m_{u}\right)+d_{v}\left(d_{v}+m_{v}\right)}{d_{u}+d_{v}}: u v \in E(G)\right\},
$$

then $G$ is a regular bipartite graph or a semiregular bipartite graph.

As an application of these lemmas, we will give some improved upper bounds for the Laplacian spectral radius and determine the corresponding extremal graphs.

Theorem 2.7. Let $G$ be a simple connected graph with $n$ vertices. Then

$$
\lambda(G) \leqslant \max \left\{d_{u}+\frac{d_{u}\left(m_{u}+\sqrt{m_{u}}\right)}{d_{u}+\sqrt{d_{u}}}: u \in V(G)\right\}
$$

the equality holds if and only if $G$ is a regular bipartite graph.

Proof. Define a diagonal matrix $D=\operatorname{diag}\left(d_{u}+\sqrt{d_{u}}: u \in V(G)\right)$, and consider the matrix $M=D^{-1} K(G) D$, similar to $K(G)$. Since $G$ is a simple connected graph, it is easy to see that $M$ is nonnegative and irreducible. The $(u, v)$-th entry of $M$ is equal to

$$
m_{u v}= \begin{cases}d_{u}, & \text { if } u=v \\ \frac{d_{v}+\sqrt{d_{v}}}{d_{u}+\sqrt{d_{u}}}, & \text { if } u \sim v \\ 0, & \text { otherwise }\end{cases}
$$

Here $u \sim v$ means that $u$ and $v$ are adjacent. Obviously, the $u$-th row sum of $M$ is

$$
R_{u}(M)=d_{u}+\sum_{u \sim v}\left(\frac{d_{v}+\sqrt{d_{v}}}{d_{u}+\sqrt{d_{u}}}\right)=d_{u}+\frac{d_{u} m_{u}}{d_{u}+\sqrt{d_{u}}}+\frac{\sum_{u \sim v} \sqrt{d_{v}}}{d_{u}+\sqrt{d_{u}}} .
$$


By the Cauchy-Schwarz inequality, we have

$$
\left(\sum_{u \sim v} \sqrt{d_{v}}\right)^{2} \leqslant d_{u} \sum_{u \sim v} d_{v}=d_{u}^{2} m_{u}
$$

Substituting (16) into (15) and simplifying the inequality, we obtain

$$
R_{u}(M) \leqslant d_{u}+\frac{d_{u}\left(m_{u}+\sqrt{m_{u}}\right)}{d_{u}+\sqrt{d_{u}}} .
$$

Then, by Lemmas 2.1 and 2.3, we have

$$
\lambda(G) \leqslant \mu(K)=\mu(M) \leqslant \max \left\{R_{u}(M) \mid u \in V(G)\right\},
$$

and (14) holds.

Now assume that the equality in (14) holds. Then $\lambda(G)=\mu(K)$. Therefore, $G$ is a bipartite graph(by Lemma 2.3). Moreover, every inequality in the above argument must be equality for each $u \in V(G)$ (by Lemma 2.1). Then by Cauchy-Schwarz inequality, we have $d_{v}=k_{u}$ for each $v \in N_{u}$, where $k_{u}$ is a constant corresponding to $u$, while the equality in (16) holds. It means that all vertices adjacent to $u$ have equal degrees for each $u \in V(G)$. Assume that $(S, T)$ is a bipartition of $V$. Without loss of generality, let $u \sim v$, with $u \in S$ (then $v \in T$ ). We first show that $d_{w}=d_{u}$ for each $w \in S$. In fact, since $G$ is connected, there exists at least a path $u v u_{1} v_{1} u_{2} v_{2} \ldots u_{m} v_{m} w$ such that $u_{i} \in S$ and $v_{i} \in T(i=1, \cdots, m)$. Then we have $d_{w}=d_{u_{m}}=\cdots=d_{u_{1}}=d_{u}=k_{v}$ by applying the above argument repeatedly. It follows that all vertices of $S$ have the same degree. Similarly, for any vertex $x \in T$, we can obtain $d_{x}=d_{v}=k_{u}$. That is to say, the degree of all vertices of $T$ are equal too.

Finally, we show that $k_{u}=k_{v}$. From Lemma 2.1 again, if the equality in (14) holds, then all row sums of $M$ are equal. In particular $R_{u}(M)=R_{v}(M)$. From (17) and the above argument, we have

$$
d_{u}+\frac{d_{u}\left(m_{u}+\sqrt{m_{u}}\right)}{d_{u}+\sqrt{d_{u}}}=d_{v}+\frac{d_{v}\left(m_{v}+\sqrt{m_{v}}\right)}{d_{v}+\sqrt{d_{v}}} .
$$

Applying $m_{u}=d_{v}=k_{u}$ and $m_{v}=d_{u}=k_{v}$ to this equation, it becomes

$$
k_{v}+\frac{k_{u} k_{v}+k_{v} \sqrt{k_{u}}}{k_{v}+\sqrt{k_{v}}}=k_{u}+\frac{k_{u} k_{v}+k_{u} \sqrt{k_{v}}}{k_{u}+\sqrt{k_{u}}} .
$$

Then we get the following equation by direct calculation

$$
\left(k_{u}+k_{v}+\sqrt{k_{u}}+\sqrt{k_{v}}\right)\left(\sqrt{k_{u}}-\sqrt{k_{v}}\right)=0 .
$$

Obviously, for any $u v \in E(G)$, the equation holds if and only if $k_{u}=k_{v}$. Summarizing the above discussion, we conclude that all vertices of $G$ have the same degree. Therefore, $G$ is a regular bipartite graph. 
Conversely, if $G$ is a regular bipartite graph, it is easy to verify that equality in (14) holds by simply calculation.

Remark 1. Clearly, $\frac{d_{u}\left(m_{u}+\sqrt{m_{u}}\right)}{d_{u}+\sqrt{d_{u}}}$ is the weighted average of $m_{u}$ and $\sqrt{d_{u} m_{u}}\left(d_{u}\right.$ and $\sqrt{d_{u}}$ are the corresponding weights). It means that the value of $\frac{d_{u}\left(m_{u}+\sqrt{m_{u}}\right)}{d_{u}+\sqrt{d_{u}}}$ is always between values of $m_{u}$ and $\sqrt{d_{u} m_{u}}$ for the same vertex $u$ of $G$. Despite of that, it is easy to show that upper bound (14) is better than upper bound (2) and (8) for some graphs. This will be illustrated in Section 3 .

Remark 2. From $\mu(K)=\mu(M)$, it is easy to prove that upper bound (14) still hold for the signless Laplacian matrix of a graph by applying Lemma 2.1 to (17) directly.

Now we provide another upper bound for $\lambda(G)$ by applying Brauer's theorem(Lemma 2.2 ), which is an improvement of upper bound (12).

For any $u v \in E(G)$, denote

$$
f(u, v)=\sqrt{\frac{\left(d_{u}+d_{v}-2\right)\left(d_{u}^{2} m_{u}+d_{v}^{2} m_{v}-2 d_{u} d_{v}\right)}{d_{u} d_{v}}} .
$$

Theorem 2.8. Let $G$ be a simple connected graph with $n$ vertices. Then

$$
\lambda(G) \leqslant 2+\sqrt{a b},
$$

where $a=\max \{f(u, v): u v \in E(G)\}$, and if $a=f(p, q)$, then $b=\max \{f(u, v): u v \in$ $E(G)-\{p q\}\}$. The equality holds if and only if $G$ is a regular bipartite graph or a semiregular bipartite graph, or a path with four vertices.

Proof. Let $U=\operatorname{diag}\left(d_{u} d_{v}: u v \in E(G)\right)$, and let $N=U^{-\frac{1}{2}} B U^{\frac{1}{2}}$, where $B$ is the adjacency matrix of the line graph $H$ of $G$. Obviously, the $(u v, p q)$-th entry of $N$ is

$$
n_{u v, p q}=\left\{\begin{array}{cl}
\frac{\sqrt{d_{p} d_{q}}}{\sqrt{d_{u} d_{v}}}, & \text { if } u v \sim p q ; \\
0, & \text { otherwise. }
\end{array}\right.
$$

Here $u v \sim p q$ means that $u v$ and $p q$ are adjacent in $H$.

Since $G$ is a connected graph, $H$ is connected too. Therefore $B$ and $N$ are nonnegative and irreducible matrices. By the Cauchy-Schwarz inequality, the $u v-t h$ row sum of $N$ satisfies

$$
\begin{aligned}
R_{u v}^{2}(N) & =\left(\sum_{u v \sim p q} \frac{\sqrt{d_{p} d_{q}}}{\sqrt{d_{u} d_{v}}}\right)^{2} \\
& \leqslant\left(d_{u}+d_{v}-2\right) \cdot \sum_{u v \sim p q} \frac{d_{p} d_{q}}{d_{u} d_{v}} \\
& =\frac{d_{u}+d_{v}-2}{d_{u} d_{v}} \cdot\left(\sum_{u \sim q} d_{u} d_{q}+\sum_{v \sim p} d_{p} d_{v}-2 d_{u} d_{v}\right) \\
& =f^{2}(u, v) .
\end{aligned}
$$


From Lemma 2.2, there exists at least one $u v \neq p q$, such that $\mu(N)$ is contained in the following oval region $S_{u v, p q}$ (in fact a circular disc):

$$
\mu(N)^{2} \leqslant R_{u v}(N) \cdot R_{p q}(N) .
$$

By the definition of $a, b$ and $f(u, v)$, we have

$$
\mu(N)^{2} \leqslant f(u, v) \cdot f(p, q) \leqslant a b .
$$

From Lemma 2.4, since $\mu(N)=\mu(B)$, we obtain that (18) holds.

Now assume that equality in (18) holds, i.e., $\lambda(G)=2+\sqrt{a b}$. It means that

$$
\lambda(G)=2+\mu(B)=2+\mu(N)=2+\sqrt{a b} .
$$

From the first equality and Lemma 2.4 we get that $G$ is bipartite. Moreover, we can assert that $\mu(N)$ is the boundary point of oval $S_{u v, p q}$ by the last equality. For any other oval region $S_{x y, s t}=\left\{z:|z|^{2} \leqslant R_{x y}(N) \cdot R_{s t}(N), x y\right.$, st $\left.\in E(G)\right\}$, we have

$$
R_{x y}(N) \cdot R_{s t}(N) \leqslant f(x, y) \cdot f(s, t) \leqslant a b=R_{u v}(N) \cdot R_{p q}(N) .
$$

Therefore, $S_{x y, s t} \subseteq S_{u v, p q}$, then $S=\bigcup_{x y \neq s t} S_{x y, s t}=S_{u v, p q}$. That is to say, $\mu(N)$ lies on the boundary of the union of all oval region of $N$.

If $N$ has two rows such that each of these rows contains at least two nonzero offdiagonal entries, then $\mu(N)$ is the boundary point of each oval $S_{x y, s t}$ (by Lemma 2.5). Thus, for any $x y, s t \in E(G)$ and $x y \neq s t$, the following holds

$$
\mu(N)=\sqrt{a b}=\sqrt{R_{x y}(N) \cdot R_{s t}(N)} .
$$

Therefore, all the row sums of $N$ are equal. Furthermore, using (20) and (21), it is easy to prove that $R_{x y}(N) \cdot R_{s t}(N)=f(x, y) \cdot f(s, t)$, then $R_{x y}(N)=f(x, y)$ for any $x y \in E(G)$. Repeating the proof of Theorem 2.7 in [5], we obtain that $G$ is a regular bipartite graph or a semiregular bipartite graph, or a path with four vertices.

If $N$ has exactly one row that contains at least two nonzero off-diagonal entries, then $B$ is permutation similar to the following matrix by the connectivity of the line graph $H$.

$$
\left(\begin{array}{cccc}
0 & 1 & \ldots & 1 \\
1 & 0 & \ldots & 0 \\
\vdots & \vdots & & \vdots \\
1 & 0 & \ldots & 0
\end{array}\right)
$$

Obviously, $H$ is a star graph and $G$ must be a path with four vertices. Otherwise, if $N$ has no rows contains more than one nonzero off-diagonal entries, $G$ is a path with two or three vertices, which are regular bipartite graph or semiregular bipartite graph, respectively. 
Conversely, it is easy to verify that the equality in (18) holds for all the graphs: regular bipartite graphs, semiregular bipartite graphs and path with four vertices.

Remark 3. Obviously, if we apply Lemma 2.1 to $R_{u v}(N) \leqslant f(u, v)$ directly, we can get the upper bound (12) which is the main result in [5]. Hence upper bound (18) is an improvement of (12). They are equivalent only if $a=b$.

It is worth noting that the upper bounds (1)-(12) are characterized by the degree and the average 2-degree of the vertices. As a matter of fact, we can also utilize other characteristics of the vertex to estimate the Laplacian spectral radius of graphs. The definition of such a new invariant of graphs is provided below.

If $u$ is a vertex of the triangle, then $u$ is incident with the triangle of a graph. Denote by $\triangle_{u}$ the number of the triangles associated with the vertex $u$. For example, see Figure.1, we have $\triangle_{u}=3$ and $\triangle_{v}=\triangle_{w}=0$.

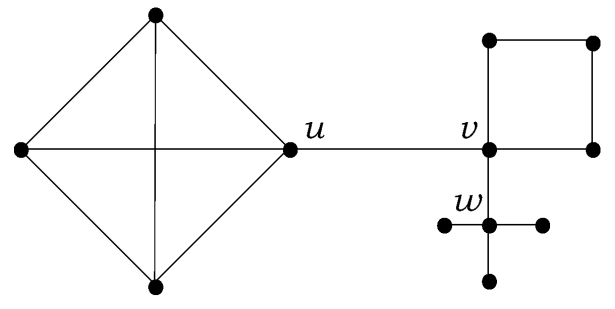

Figure.1

However, if $G$ is a bipartite graph, then it does not contain any odd cycle, hence $\triangle_{u}$ is always zero for any $u \in V(G)$.

Theorem 2.9. Let $G$ be a simple connected graph with $n$ vertices. Then

$$
\lambda(G) \leqslant \max \left\{\frac{d_{u}\left(d_{u}+m_{u}\right)+d_{v}\left(d_{v}+m_{v}\right)-2\left(\triangle_{u}+\triangle_{v}\right)}{d_{u}+d_{v}-\left|N_{u} \cap N_{v}\right|}: u v \in E(G)\right\} .
$$

The equality holds if and only if $G$ is a regular bipartite graph or a semiregular bipartite graph.

Proof. Let $V=\operatorname{diag}\left(d_{u}+d_{v}-\left|N_{u} \cap N_{v}\right|: u v \in E(G)\right)$, where $\left|N_{u} \cap N_{v}\right|$ is the number of common neighbors of vertices $u$ and $v$. Consider the matrix $N=V^{-1} B V$, similar to $B$. Obviously, the $(u v, p q)$-th entry of $N$ is equal to

$$
n_{u v, p q}= \begin{cases}\frac{d_{p}+d_{q}-\left|N_{p} \cap N_{q}\right|}{d_{u}+d_{v}-\left|N_{u} \cap N_{v}\right|}, & \text { if } u v \sim p q \\ 0, & \text { otherwise }\end{cases}
$$

Since $G$ is connected graph, $B$ and $N$ are nonnegative and irreducible. Then the $u v-t h$ row sum of $N$ is 


$$
\begin{aligned}
R_{u v}(N)= & \sum_{u v \sim p q}\left(\frac{d_{p}+d_{q}-\left|N_{p} \cap N_{q}\right|}{d_{u}+d_{v}-\left|N_{u} \cap N_{v}\right|}\right) \\
= & \frac{\sum_{u \sim q}\left(d_{u}+d_{q}\right)+\sum_{v \sim p}\left(d_{p}+d_{v}\right)-2\left(d_{u}+d_{v}\right)}{d_{u}+d_{v}-\left|N_{u} \cap N_{v}\right|} \\
& \quad-\frac{\sum_{u \sim q}\left|N_{u} \cap N_{q}\right|+\sum_{v \sim p}\left|N_{p} \cap N_{v}\right|-2\left|N_{u} \cap N_{v}\right|}{d_{u}+d_{v}-\left|N_{u} \cap N_{v}\right|} \\
= & \frac{d_{u}\left(d_{u}+m_{u}\right)+d_{v}\left(d_{v}+m_{v}\right)-2\left(d_{u}+d_{v}\right)}{d_{u}+d_{v}-\left|N_{u} \cap N_{v}\right|} \\
& -\frac{2\left(\triangle_{u}+\triangle_{v}\right)-2\left|N_{u} \cap N_{v}\right|}{d_{u}+d_{v}-\left|N_{u} \cap N_{v}\right|} \\
= & \frac{d_{u}\left(d_{u}+m_{u}\right)+d_{v}\left(d_{v}+m_{v}\right)-2\left(\triangle_{u}+\triangle_{v}\right)}{d_{u}+d_{v}-\left|N_{u} \cap N_{v}\right|}-2 .
\end{aligned}
$$

Since $\mu(N)=\mu(B)$, from Lemmas 2.1 and 2.4, (22) holds.

If the equality in (22) holds, $G$ is a bipartite graph (by Lemma 2.4). Thus $G$ has no odd cycles, which means that $\triangle_{u}=\triangle_{v}=0$ and $N_{u} \cap N_{v}=\phi$ for any $u v \in E(G)$. Therefore, (22) becomes

$$
\lambda(G)=\max \left\{\frac{d_{u}\left(d_{u}+m_{u}\right)+d_{v}\left(d_{v}+m_{v}\right)}{d_{u}+d_{v}}: u v \in E(G)\right\} .
$$

Then it follows from Lemma 2.6 that $G$ is bipartite regular or semi-regular.

Finally, if $G$ is a regular bipartite graph or a semiregular bipartite graph, it is easy to verify, by simple calculation, that equality in (22) holds.

Remark 4. If $G$ is a bipartite graph, then it follows from Lemma 2.3 that $\lambda(G)=$ $\mu(K)$. Therefore, upper bounds (18) and (22) still hold for the signless Laplacian matrix of a bipartite graph.

\section{$3 \quad$ Examples}

In this section, three examples are presented to illustrate that (14), (18) and (22) are better than other upper bounds in some sense.

Example. Let $G_{1}, G_{2}$ and $G_{3}$ be the graphs of order 30, 91 and 11, respectively, as shown in Figure.2. Clearly, $G_{2}$ and $G_{3}$ are trees. 

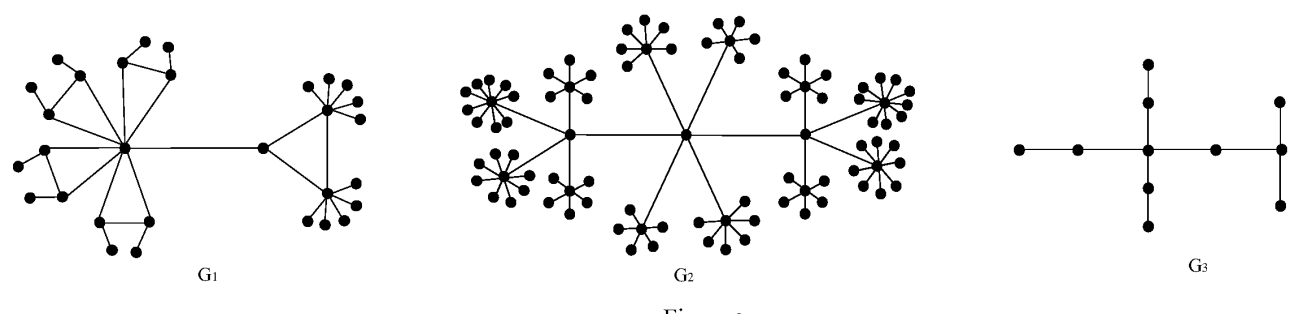

Figure.2

We summarize all classic upper bounds for the largest Laplacian eigenvalue of $G_{1}, G_{2}$ and $G_{3}$ as follows:

Table 1: The value of all known upper bounds for the examples

\begin{tabular}{cccccccccccc}
\hline & $(1)$ & $(3)$ & $(6)$ & $(7)$ & $(8)$ & $(9)$ & $(10)$ & $(11)$ & $(14)$ & $(18)$ & $(22)$ \\
\hline$G_{1}$ & 12.96 & 11.67 & 13 & 11.66 & 14.20 & 11.83 & 12.13 & 11.82 & 12.55 & 11.48 & 10.91 \\
$G_{2}$ & 15 & 12.18 & 15 & 12.18 & 13.74 & 13.27 & 12.28 & 12.88 & 12 & 12.82 & 12.18 \\
$G_{3}$ & 6 & 5.83 & 6 & 5.83 & 6.83 & 5.92 & 6.47 & 5.91 & 6.27 & 5.74 & 5.83 \\
\hline
\end{tabular}

Remark 5. Obviously, from Table 1, bound (14) is the best in all known upper bounds for $G_{2}$, and bound (18) is the best in all known upper bounds for $G_{3}$. Finally, bound (22) is the best upper bound for $G_{1}$ and bound (18) is the second-best except bound (22) for $G_{1}$. Of course, bound (22) and (3) are equivalent for $G_{2}$ and $G_{3}$, because they are trees. In general, these upper bounds are incomparable.

\section{Acknowledgements.}

The authors would like to thank the anonymous referees for their remarks and suggestions which greatly improved the presentation of this paper.

This work is supported by the National Natural Science Foundation of China (Grant No.61003310) and the Research Fund of Sichuan Provincial Education Department (Grant No.07ZB031).

\section{References}

[1] R. Merris, Laplacian Matrices of Graphs: A survey, Linear Algebra Appl., 197-198(1994), 143-176.

[2] B. Mohar, Some applications of Laplace eigenvalues of graphs, Graph Symmetry (G.Harn and G.Sabiussi, Eds.), Dordrecht: Kluwer Academic Publishers, 1997.

[3] F. Chung, The diameter and Laplacian eigenvalues of directed graphs, Electron. J. Combin. 13(2006), N4.

[4] S. Kirkland, Laplacian Integral Graphs with Maximum Degree 3, Electron. J. Combin. 15(2008), R120.

[5] T.F. Wang, Several sharp upper bounds for the largest laplacian eigenvalue of a graph, Science in China Series A: mathematics, 12(2007), 1000-1008. 
[6] J.S. Li, X.D. Zhang, A new upper bound for eigenvalues of the Laplacian Matrix of a graph, Linear Algebra Appl., 265(1997), 93-100.

[7] R. Merris, A note on Laplacian graph eigenvalues, Linear Algebra Appl., 285(1998), 33-35.

[8] J.S. Li, X.D. Zhang, On the Laplacian eigenvalues of a graph, Linear Algebra Appl., 285(1998), 305-307.

[9] O. Rojo,R. Soto,H. Rojo, An always nontrivial upper bound for Laplacian graph eigenvalues, Linear Algebra Appl., 312(2000), 155-159.

[10] J.S. Li, Y.L. Pan, De Caen's inequality and bounds on the largest Laplacian eigenvalue of a graph, Linear Algebra Appl., 328(2001), 153-160.

[11] K.C. Das, An improved upper bound for Laplacian graph eigenvalues, Linear Algebra Appl. , 368(2003), 269-278.

[12] K.C. Das, A characterization on graphs which achieve the upper bound for the largest Laplacian eigenvalue of graphs, Linear Algebra Appl., 376(2004), 173-186.

[13] X.D. Zhang, Two sharp upper bounds for the Laplacian eigenvalues, Linear Algebra Appl., 376(2004), 207-213.

[14] J.M. Guo, A new upper bound for the Laplacian spectral radius of graphs, Linear Algebra Appl., 400(2005), 61-66.

[15] R.A. Horn, C.R. Johnson, Matrix Analysis, New York: Cambridge University Press, 1985.

[16] Y.L. Pan, Sharp upper bounds for the Laplacian graph eigenvalues, Linear Algebra Appl., $355(2002)$, 287-295.

[17] J.L. Shu, Y. Hong, R.K. Wen, A Sharp upper bound on the largest eigenvalue of the Laplacian matrix of a graph, Linear Algebra Appl., 347(2002), 123-129. 